\title{
The Strong Sequential Core in a Dynamic Exchange Economy
}

Citation for published version (APA):

Predtetchinski, A., Herings, P. J. J., \& Peters, H. J. M. (2004). The Strong Sequential Core in a Dynamic Exchange Economy. Economic Theory, 24, 147-162. https://doi.org/10.1007/s00199-003-0404-1

Document status and date:

Published: 01/01/2004

DOI:

10.1007/s00199-003-0404-1

Document Version:

Publisher's PDF, also known as Version of record

\section{Please check the document version of this publication:}

- A submitted manuscript is the version of the article upon submission and before peer-review. There can be important differences between the submitted version and the official published version of record.

People interested in the research are advised to contact the author for the final version of the publication, or visit the DOI to the publisher's website.

- The final author version and the galley proof are versions of the publication after peer review.

- The final published version features the final layout of the paper including the volume, issue and page numbers.

Link to publication

\footnotetext{
General rights rights.

- You may freely distribute the URL identifying the publication in the public portal. please follow below link for the End User Agreement:

www.umlib.nl/taverne-license

Take down policy

If you believe that this document breaches copyright please contact us at:

repository@maastrichtuniversity.nl

providing details and we will investigate your claim.
}

Copyright and moral rights for the publications made accessible in the public portal are retained by the authors and/or other copyright owners and it is a condition of accessing publications that users recognise and abide by the legal requirements associated with these

- Users may download and print one copy of any publication from the public portal for the purpose of private study or research.

- You may not further distribute the material or use it for any profit-making activity or commercial gain

If the publication is distributed under the terms of Article $25 \mathrm{fa}$ of the Dutch Copyright Act, indicated by the "Taverne" license above, 


\title{
The strong sequential core in a dynamic exchange economy ${ }^{\star}$
}

\author{
Arkadi Predtetchinski ${ }^{1}$, P. Jean-Jacques Herings ${ }^{1}$, and Hans Peters ${ }^{2}$ \\ 1 Department of Economics, Universiteit Maastricht, P.O. Box 616, \\ 6200 MD Maastricht, THE NETHERLANDS \\ (e-mail: \{a.predtetchinski;P.Herings\} @ algec.unimaas.nl) \\ 2 Department of Quantitative Economics, Universiteit Maastricht, P.O. Box 616, \\ 6200 MD Maastricht, THE NETHERLANDS \\ (e-mail: h.peters@ke.unimaas.nl)
}

Received: August 22, 2002; revised version: May 22, 2003

Summary. Dynamic exchange economies with uncertainty are considered where the information is released over infinite time. The strong sequential core of such an economy consists of those consumption streams that can be improved upon by no coalition at no moment of time. Non-emptiness of the strong sequential core is established given a high enough discount factor. Moreover, sufficient conditions are given under which the strong sequential core contains only time and history independent consumption streams.

Keywords and Phrases: Core, Stationary economies, Uncertainty.

JEL Classification Numbers: C71, C73, D51, D52.

\section{Introduction}

One of the major difficulties in modeling cooperation in a dynamic environment is the potential abundance of opportunities for coalitions to deviate from earlier agreements. As the initial positions of agents and coalitions and their preferences may change over time, a contract that was originally considered acceptable may no longer be so after some time. Thus, agents or coalitions may tend to break agreements reached earlier in the game. In the case of uncertainty, where information becomes available over time, this is even more likely to occur. What was perceived

\footnotetext{
* Jean-Jacques Herings would like to thank the Netherlands Organization for Scientific Research for financial support.

Correspondence to: P. J. J. Herings
} 
as an acceptable agreement when no information was available, may no longer be so after some information has been released.

In a dynamic economy the classical core concept as introduced in Aumann (1961) is still meaningful. A consumption stream is an element of the classical core if no coalition can make its members better off by pooling their own resources and deviating at period zero for the rest of the time. The classical core, however, ignores incentives for coalitional improvement that may arise in subsequent periods. It is essentially a static concept, for it does not depend on the time or the information structure of the economy.

These considerations motivate the search for a core concept that offers a more appropriate view on cooperation taking place in a dynamic, rather than a static environment. Koutsougeras and Yannelis (1999) and Serfes (2001) consider the concepts of the private core and the non-myopic core in dynamic differential information economies. They study the evolution of core allocations, as the agents acquire more information through time. To the best of our knowledge, the concept of the so-called sequential core was first proposed in Gale (1978) in a model of a monetary economy. Other core concepts that take into account the dynamic structure of the environment have been proposed in Repullo (1988), Koutsougeras (1998), and Filar and Petrosjan (2000). Kranich, Perea, and Peters (2001) distinguish between a strong and a weak version of the sequential core. They study both concepts in a deterministic setting where agents face a finite sequence of transferable utility games. In Predtetchinski, Herings, and Peters (2002) the notion of the strong sequential core is applied to an economy with two periods and uncertainty where agents are given a possibility to exchange assets in the first period.

Despite the differences in the models, the mentioned papers of Gale (1978), Kranich et al. (2001), and Predtetchinski et al. (2001) share a common approach to the definition of the strong sequential core. The dynamic nature of the economy allows for a number of sub-economies to be identified. The consumption stream is then said to be a strong sequential core-element if it belongs to the classical core of each of the sub-economies of the original dynamic economy. Hence, at no particular moment can a coalition improve by deviating for the rest of time.

In Predtetchinski, Herings, and Peters (2002) the strong sequential core is defined in such a way that, as a set, it is weakly increasing in the number of assets. In this sense, the presence of assets can potentially lead to non-emptiness of the strong sequential core. Nevertheless, a number of negative results is established even in this context. Generic emptiness is shown in the setting of a finance economy with incomplete markets, and an example of emptiness is presented even for asset structures satisfying a very strong condition of completeness. The general conclusion is that, unless a complete set of state-contingent contracts is available for trade, non-emptiness of the strong sequential core cannot be guaranteed.

These negative results provide a motivation to augment the basic model in a different direction, namely to consider a dynamic exchange economy with an infinite time horizon. At each moment of time the economy is in one of a finite number of states. The state determines the consumption sets, the instantaneous utility functions, and the initial endowments of all agents at that moment of time. Transition from one state to the next occurs according to a given Markov process. 
The main result of the paper states that the strong sequential core of a dynamic exchange economy is non-empty, provided that the discount factor that agents apply to future utility streams is sufficiently close to one.

In more detail, the content of the paper is as follows. In Section 2 a dynamic exchange economy is introduced, and the concepts of the classical core and of the strong sequential core are discussed. In Section 3 a characterization of the strong sequential core is developed. We show that the classical core consists only of time and history independent consumption streams. This property of the core allows for a representation of a dynamic sub-economy by means of a finite-dimensional static evaluation economy. We prove that the strong sequential core is non-empty if and only if cores of the evaluation economies have a non-empty intersection. This result should be seen as closely related to the idea of a value function in dynamic programming. Section 4 presents an example illustrating a non-emptiness criterion for the strong sequential core. Section 5 provides a non-emptiness result for the strong sequential core. More precisely, this result says that a competitive equilibrium allocation of a specific limit economy is in the strong sequential core whenever the discount factor is sufficiently close to one. Section 6 concludes.

\section{Dynamic exchange economies}

A dynamic exchange economy is given by the tuple

$$
\mathbf{E}=\left\langle N, \mathbf{S}, L,\left(X_{s}^{i}, u_{s}^{i}, e_{s}^{i}\right)_{s \in \mathbf{S}}^{i \in N}, \pi, \delta\right\rangle .
$$

The ingredients of a dynamic exchange economy $\mathbf{E}$ are as follows. The set $N=$ $\{1, \ldots, n\}$ is the set of agents and $\mathbf{S}=\{1, \ldots, S\}$ is the set of states. The symbol $L$ denotes the number of consumption goods in each state and each time period. The symbols $X_{s}^{i}, u_{s}^{i}$, and $e_{s}^{i}$ denote, respectively, the consumption set, the instantaneous utility function, and the vector of initial endowments of agent $i \in N$ in state $s \in \mathbf{S}$. The matrix $\pi=(\pi(\sigma \mid s))_{\sigma, s \in \mathbf{S}}$ is a column stochastic matrix of transitional probabilities: for any $\sigma$ and $s$ in $\mathbf{S}, \pi(\sigma \mid s)$ is the transition probability from state $s$ to state $\sigma$. We assume that the agents' preferences over consumption streams can be represented by the expected discounted utility function. The discount factor is given by $\delta$.

The basic assumption of the model is the following:

\section{Assumption A.}

(A1) For all $i \in N$ and $s \in \mathbf{S}$ the consumption set $X_{s}^{i}$ is a non-empty, convex, closed, bounded from below subset of $\mathbb{R}^{L}$.

(A2) For all $i \in N$ and $s \in \mathbf{S}$ the instantaneous utility function $u_{s}^{i}: X_{s}^{i} \rightarrow \mathbb{R}$ is continuous and concave.

(A3) For all $i \in N$ and $s \in \mathbf{S}$, the vector of initial endowments $e_{s}^{i}$ is an element of the consumption set $X_{s}^{i}$.

(A4) The matrix $\pi$ is irreducible.

(A5) $\delta \in(0,1)$. 


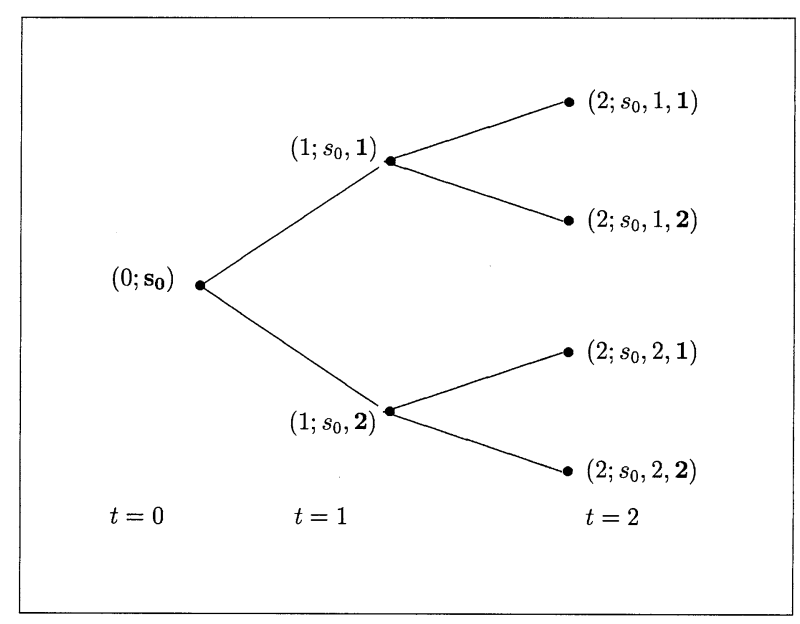

Figure 1. A date-event tree with $S=2$

A dynamic exchange economy is an economy with infinite time horizon, so the set of time periods or dates is $\mathbf{T}=\{0,1, \ldots t, \ldots\}$. At each date $t \in \mathbf{T}$ the state takes on one of the values from the set $\mathbf{S}$ determining the consumption sets, the instantaneous utility functions, and the initial endowments of all agents at date $t$. The state follows a Markov process with one-step transitional probabilities given by $\pi$. At date $t=0$ the state is known to be $s_{0} \in \mathbf{S}$.

A date-event tree describes the set of all contingencies that may potentially arise during the course of the game. Formally, it is the set $\mathbb{D}$ defined as follows:

$$
\mathbb{D}=\left\{\left(t, s_{0}, s_{1}, \ldots, s_{t}\right) \mid t \in \mathbf{T}, s_{\tau} \in \mathbf{S} \text { for all } \tau \in\{1, \ldots t\}\right\}
$$

The elements of the date-event tree are called nodes. Node $\xi=\left(t, s_{0}, s_{1}, \ldots, s_{t}\right)$ of the date-event tree summarizes the information available to agents at time $t$. This information includes the current date $t$ at the node $\xi$, the history $\left(s_{0}, s_{1}, \ldots, s_{t-1}\right)$ of the state of nature preceding date $t$, and the current state $s_{t}$ at node $\xi$. The mapping $t: \mathbb{D} \rightarrow \mathbf{T}$ assigns to each node $\xi \in \mathbb{D}$ its current date $t(\xi)$. The mapping $s: \mathbb{D} \rightarrow \mathbf{S}$ assigns to node $\xi$ its current state $s(\xi)$.

For the case $S=2$ the date-event tree is partially reproduced in Figure 1 . Numbers in bold type indicate the current states.

Node $\xi=\left(t, s_{0}, s_{1}, \ldots, s_{t}\right)$ of the date-event tree gives rise to a subtree $\mathbb{D}(\xi)$ defined as follows:

$$
\mathbb{D}(\xi)=\left\{\left(\tau, \sigma_{0}, \sigma_{1}, \ldots, \sigma_{\tau}\right) \in \mathbb{D} \mid \tau \geq t, \sigma_{k}=s_{k} \text { for all } k \in\{0,1, \ldots, t\}\right\}
$$

The probability that the node $\eta=\left(\tau, \sigma_{0}, \sigma_{1}, \ldots, \sigma_{\tau}\right)$ will be reached conditional on the fact that the node $\xi=\left(t, s_{0}, s_{1}, \ldots, s_{t}\right)$ has been reached is defined by the 
following formula:

$$
\rho(\eta \mid \xi)= \begin{cases}1, & \text { if } \eta=\xi \\ \prod_{k=t+1}^{\tau} \pi\left(\sigma_{k} \mid \sigma_{k-1}\right), & \text { if } \eta \in \mathbb{D}(\xi) \text { and } \tau>t \\ 0, & \text { otherwise. }\end{cases}
$$

A mapping $\mathcal{X}^{i}: \mathbb{D} \rightarrow \mathbb{R}^{L}$ is called the consumption stream of agent $i \in N$ if its value $\mathcal{X}_{\xi}^{i}$ at every node $\xi$ of the date-event tree belongs to the consumption set $X_{s(\xi)}^{i}$ of agent $i$. The collection of the consumption streams $\mathcal{X}=\left\langle\mathcal{X}^{i}\right\rangle_{i \in Q}$ is called a commodity allocation for the coalition of agents $Q \subseteq N$.

The expected discounted utility of the consumption stream $\mathcal{X}^{i}$ of agent $i$ at the node $\xi$ is given by the function

$$
\mathcal{V}_{\mathbb{D}(\xi)}^{i}\left(\mathcal{X}^{i}\right)=\sum_{\eta \in \mathbb{D}(\xi)} \rho(\eta \mid \xi) \delta^{t(\eta)-t(\xi)} u_{s(\eta)}^{i}\left(\mathcal{X}_{\eta}^{i}\right)
$$

Given a dynamic exchange economy $\mathbf{E}$ and a node $\xi$ of the date-event tree, let $\mathbf{E}_{\mathbb{D}(\xi)}$ denote the subeconomy of $\mathbf{E}$ starting at the node $\xi$.

We continue with the definitions of the classical core for the subeconomy $\mathbf{E}_{\mathbb{D}(\xi)}$ and of the strong sequential core for the economy E. Essentially, the former definition requires that no coalition be able to make all its members better off by deviating at node $\xi$ for the rest of time. The latter definition requires that no coalition be able to improve by deviating at any of the nodes of the date-event tree. In this way, the strong sequential core eliminates any incentives for coalitions to first agree to a proposed allocation but break the agreement later on.

Definition 1. A commodity allocation $\mathcal{X}=\left\langle\mathcal{X}^{i}\right\rangle_{i \in N}$ belongs to the classical core $C\left(\mathbf{E}_{\mathbb{D}(\xi)}\right)$ of the sub-economy $\mathbf{E}_{\mathbb{D}(\xi)}$ if

1. $\sum_{i \in N} \mathcal{X}_{\eta}^{i}=\sum_{i \in N} e_{s(\eta)}^{i}$ for all $\eta \in \mathbb{D}(\xi)$,

2. there exist no coalition $Q \subseteq N$ and no commodity allocation $\mathcal{Y}=\left\langle\mathcal{Y}^{i}\right\rangle_{i \in Q}$ such that $\sum_{i \in Q} \mathcal{Y}_{\eta}^{i}=\sum_{i \in Q} e_{s(\eta)}^{i}$ for all $\eta \in \mathbb{D}(\xi)$ and $\mathcal{V}_{\mathbb{D}(\xi)}^{i}\left(\mathcal{Y}^{i}\right)>\mathcal{V}_{\mathbb{D}(\xi)}^{i}\left(\mathcal{X}^{i}\right)$ for all $i \in Q$.

Definition 2. The strong sequential core of the dynamic exchange economy $\mathbf{E}$ is the set of commodity allocations

$$
S S C(\mathbf{E}):=\bigcap_{\xi \in \mathbb{D}} C\left(\mathbf{E}_{\mathbb{D}(\xi)}\right) .
$$

\section{A characterization of the strong sequential core}

An important role in the characterization of the strong sequential core is played by time and history independent consumption streams. Consumption stream $\mathcal{X}^{i}$ of agent $i$ is said to be time and history independent if the value for $\mathcal{X}_{\xi}^{i}$ depends on the current state $s(\xi)$ only. Given an element $x^{i}$ of the set $\prod_{\sigma \in \mathbf{S}} X_{\sigma}^{i}$, let $\left\{x^{i}\right\}$ denote the time and history independent consumption stream $\mathcal{X}^{i}: \mathbb{D} \rightarrow \mathbb{R}^{L}$ defined 
by $\mathcal{X}_{\xi}^{i}=x_{s(\xi)}^{i}$ for all $\xi \in \mathbb{D}$. Let $\{x\}$ denote the time and history independent commodity allocation $\left\langle\left\{x^{i}\right\}\right\rangle_{i \in N}$.

The evaluation economies constitute another important aspect of the characterization of the strong sequential core. Let $I_{S}$ denote the $S$-dimensional identity matrix. Observe that the matrix $I_{S}-\delta \pi$ is invertible, since the spectral radius of the matrix $\delta \pi$ equals $\delta<1$. Let $\psi=\left(I_{S}-\delta \pi\right)^{-1}$, and for every pair of states $\sigma$ and $s$ let $\psi(\sigma \mid s)$ denote an element of the matrix $\psi$ at the intersection of row $\sigma$ and column $s$. If the matrix $\pi$ is irreducible, then $\psi(\sigma \mid s)>0$ for all $\sigma, s \in \mathbf{S}$.

Definition 3. The evaluation economy $\mathcal{E}_{s}$ in state $s$ is the pure exchange economy where $\mathbb{R}^{S L}$ is the commodity space, $X^{i}=\prod_{\sigma \in \mathbf{S}} X_{\sigma}^{i}$ is agent $i$ 's consumption set, $e^{i}=\left(e_{\sigma}^{i}\right)_{\sigma \in \mathbf{S}}$ is the vector of initial endowments, and $v_{s}^{i}: X^{i} \rightarrow \mathbb{R}$ is the utility function defined by

$$
v_{s}^{i}\left(x^{i}\right)=\sum_{\sigma \in \mathbf{S}} \psi(\sigma \mid s) u_{\sigma}^{i}\left(x_{\sigma}^{i}\right) .
$$

Lemma 1 below clarifies the role of the evaluation economies in our analysis. The economy $\mathcal{E}_{s}$ can be thought of as a static ("normal form") representation of the subeconomy $\mathbf{E}_{\mathbb{D}(\xi)}$ starting at the node $\xi$ with current state $s$.

Lemma 1. $\mathcal{V}_{\mathbb{D}(\xi)}^{i}\left(\left\{x^{i}\right\}\right)=v_{s(\xi)}^{i}\left(x^{i}\right)$ for all $\xi \in \mathbb{D}$ and $x^{i} \in X^{i}$.

Proof. Let $\xi \in \mathbb{D}$ be given. First we argue that

$$
\psi(\sigma \mid s(\xi))=\sum_{\substack{\eta \in \mathbb{D}(\xi) \\ s(\eta)=\sigma}} \rho(\eta \mid \xi) \delta^{t(\eta)-t(\xi)} .
$$

Indeed, the right-hand side of equation (2) can be written as

$$
\sum_{\tau \in \mathbf{T}} \delta^{\tau}\left[\sum_{\eta} \rho(\eta \mid \xi)\right],
$$

where the sum in square brackets is taken over all nodes $\eta \in \mathbb{D}(\xi)$ such that $t(\eta)=$ $t(\xi)+\tau$ and $s(\eta)=\sigma$. The expression in square brackets gives the probability that state $\sigma$ will be reached from state $s(\xi)$ in exactly $\tau$ periods. It is therefore equal to the entry of the matrix $\pi^{\tau}$ at the intersection of row $\sigma$ and column $s(\xi)$. Hence, the right-hand side of equation (2) equals the entry of the matrix $\sum_{\tau \in \mathbf{T}} \delta^{\tau} \pi^{\tau}$ at the intersection of row $\sigma$ and column $s(\xi)$. Finally, observe that $\sum_{\tau \in \mathbf{T}} \delta^{\tau} \pi^{\tau}=\psi$.

Given $x^{i} \in X^{i}$, the expected discounted utility of the consumption stream $\left\{x^{i}\right\}$ at node $\xi$ can be written as

$$
\begin{aligned}
\mathcal{V}_{\mathbb{D}(\xi)}^{i}\left(\left\{x^{i}\right\}\right) & =\sum_{\sigma \in \mathbf{S}} \sum_{\substack{\eta \in \mathbb{D}(\xi) \\
s(\eta)=\sigma}} \rho(\eta \mid \xi) \delta^{t(\eta)-t(\xi)} u_{\sigma}^{i}\left(x_{\sigma}^{i}\right) \\
& =\sum_{\sigma \in \mathbf{S}} \psi(\sigma \mid s(\xi)) u_{\sigma}^{i}\left(x_{\sigma}^{i}\right) \\
& =v_{s(\xi)}^{i}\left(x^{i}\right) .
\end{aligned}
$$


Theorem 1 provides a necessary and sufficient condition for a given time and history independent commodity allocation to be an element of the strong sequential core.

Theorem 1. Let $\mathbf{E}$ be a dynamic exchange economy satisfying assumption $\mathbf{A}$. Let $\mathcal{X}=\{x\}$ be a time and history independent commodity allocation. Then

1. $\mathcal{X} \in C\left(\mathbf{E}_{\mathbb{D}(\xi)}\right)$ if and only if $x \in C\left(\mathcal{E}_{s(\xi)}\right)$.

2. $\mathcal{X} \in S S C(\mathbf{E})$ if and only if $x \in C\left(\mathcal{E}_{s}\right)$ for all $s \in \mathbf{S}$.

Proof. Let the time and history independent commodity allocation $\mathcal{X}=\{x\}$ be given. To prove the first part of Theorem 1 we demonstrate that a coalition $Q \subseteq N$ is able to improve upon $\mathcal{X}$ in the subeconomy $\mathbf{E}_{\mathbb{D}(\xi)}$ if and only if it can improve upon $x$ in the evaluation economy $\mathcal{E}_{s(\xi)}$. The second part of Theorem 1 is an immediate corollary of the first part.

Suppose that there is a deviation $y$ from $x$ by a coalition $Q$ in the evaluation economy $\mathcal{E}_{s(\xi)}$. Lemma 1 implies that the time and history independent commodity allocation $\{y\}$ is a deviation from the allocation $\{x\}$ in the subeconomy $\mathbf{E}_{\mathbb{D}(\xi)}$.

Suppose that there is a deviation $\mathcal{Y}$ from the allocation $\{x\}$ by a coalition $Q$ in the subeconomy $\mathbf{E}_{\mathbb{D}(\xi)}$. Note that the allocation $\mathcal{Y}$ need not be time and history independent. In the remainder of the proof we show that there does exist a time and history independent allocation $\mathcal{Z}=\{z\}$ that (a) is feasible for $Q$ and (b) gives each member of the coalition $Q$ at least as high expected discounted utility at node $\xi$ as $\mathcal{Y}$ does. An allocation $\mathcal{Z}$ having these properties is a deviation from the allocation $\{x\}$ in the subeconomy $\mathbf{E}_{\mathbb{D}(\xi)}$. By Lemma $1, z$ is a deviation from $x$ in the evaluation economy $\mathcal{E}_{s(\xi)}$.

Define $z$ by

$$
z_{\sigma}^{i}=\frac{1}{\psi(\sigma \mid s(\xi))} \sum_{\substack{\eta \in \mathbb{D}(\xi) \\ s(\eta)=\sigma}} \rho(\eta \mid \xi) \delta^{t(\eta)-t(\xi)} \mathcal{Y}_{\eta}^{i},
$$

for all $i \in Q$ and $\sigma \in \mathbf{S}$. As the allocation $\mathcal{Y}$ is feasible for coalition $Q$, and the consumption sets $X_{\sigma}^{i}$ are assumed to be bounded from below for all $i \in Q$, the consumption stream $\mathcal{Y}^{i}$ is bounded. Hence, the sum in equation (3) is welldefined. Equation (2) implies that $z_{\sigma}^{i}$ is a convex combination of the values for the consumption stream $\mathcal{Y}^{i}$ at all nodes $\eta$ in the subtree $\mathbb{D}(\xi)$ with $s(\eta)=\sigma$. For all such nodes, $\mathcal{Y}_{\eta}^{i} \in X_{\sigma}^{i}$. Since the set $X_{\sigma}^{i}$ is assumed to be closed and convex, $z_{\sigma}^{i} \in X_{\sigma}^{i}$. Moreover, $z$ is feasible for $Q$. Continuity and concavity of the instantaneous utility functions imply the inequalities

$$
u_{\sigma}^{i}\left(z_{\sigma}^{i}\right) \geq \frac{1}{\psi(\sigma \mid s(\xi))} \sum_{\substack{\eta \in \mathbb{D}(\xi) \\ s(\eta)=\sigma}} \rho(\eta \mid \xi) \delta^{t(\eta)-t(\xi)} u_{\sigma}^{i}\left(\mathcal{Y}_{\eta}^{i}\right),
$$


for all $i \in Q, \sigma \in \mathbf{S}$. By Lemma 1 , the discounted expected utility of the consumption stream $\mathcal{Z}^{i}=\left\{z^{i}\right\}$ is

$$
\begin{aligned}
\mathcal{V}_{\mathbb{D}(\xi)}^{i}\left(\mathcal{Z}^{i}\right) & =\sum_{\sigma \in \mathbf{S}} \psi(\sigma \mid s(\xi)) u_{\sigma}^{i}\left(z_{\sigma}^{i}\right) \\
& \geq \sum_{\sigma \in \mathbf{S}} \sum_{\substack{\eta \in \mathbb{D}(\xi) \\
s(\eta)=\sigma}} \rho(\eta \mid \xi) \delta^{t(\eta)-t(\xi)} u_{\sigma}^{i}\left(\mathcal{Y}_{\eta}^{i}\right) \\
& =\sum_{\eta \in \mathbb{D}(\xi)} \rho(\eta \mid \xi) \delta^{t(\eta)-t(\xi)} u_{s(\eta)}^{i}\left(\mathcal{Y}_{\eta}^{i}\right) \\
& =\mathcal{V}_{\mathbb{D}(\xi)}^{i}\left(\mathcal{Y}^{i}\right)
\end{aligned}
$$

for all $i \in Q$.

Theorem 2 gives sufficient conditions for the classical core to contain only time and history independent commodity allocations.

Theorem 2. Let $\mathbf{E}$ be a dynamic exchange economy satisfying assumption Ain which $\pi(\sigma \mid s)>0$ for all $\sigma, s \in \mathbf{S}, X_{\sigma}^{i}=\mathbb{R}_{+}^{L}, u_{\sigma}^{i}$ are strongly monotone ${ }^{1}$ for all $i \in N, \sigma \in \mathbf{S}$ and strictly concave for all $i \in\{1, \ldots, n-1\}, \sigma \in \mathbf{S}$. Then the classical core of the economy $\mathbf{E}$ contains no time or history dependent commodity allocations.

Proof. Let $\mathcal{X}$ be an $N$-feasible commodity allocation. If the allocation $\mathcal{X}$ is not time and history independent, then there exists a pair of nodes $\lambda, \varsigma \in \mathbb{D}$ with the same current state $\sigma$ such that $\mathcal{X}_{\lambda}^{i_{0}} \neq \mathcal{X}_{\varsigma}^{i_{0}}$ for some $i_{0} \in N$. Observe that both $\mathcal{X}_{\lambda}^{i}$ and $\mathcal{X}_{\varsigma}^{i}$ are elements of the consumption set $X_{\sigma}^{i}$ for all $i \in N$.

Let $\rho(\lambda)$ and $\rho(\varsigma)$ be probabilities to reach nodes $\lambda$ and $\varsigma$ conditional on the initial node $\left(0, s_{0}\right)$. Under the assumption that all transition probabilities are positive, $\rho(\lambda)$ and $\rho(\varsigma)$ are both positive. Define an element $\widetilde{y}_{\sigma}^{i}$ of the set $X_{\sigma}^{i}$ as

$$
\widetilde{y}_{\sigma}^{i}=\frac{\delta^{t(\lambda)} \rho(\lambda) \mathcal{X}_{\lambda}^{i}+\delta^{t(\varsigma)} \rho(\varsigma) \mathcal{X}_{\varsigma}^{i}}{\delta^{t(\lambda)} \rho(\lambda)+\delta^{t(\varsigma)} \rho(\varsigma)} .
$$

By concavity of the instantaneous utility functions,

$$
u_{\sigma}^{i}\left(\widetilde{y}_{\sigma}^{i}\right) \geq \frac{\delta^{t(\lambda)} \rho(\lambda) u_{\sigma}^{i}\left(\mathcal{X}_{\lambda}^{i}\right)+\delta^{t(\varsigma)} \rho(\varsigma) u_{\sigma}^{i}\left(\mathcal{X}_{\varsigma}^{i}\right)}{\delta^{t(\lambda)} \rho(\lambda)+\delta^{t(\varsigma)} \rho(\varsigma)} .
$$

for all $i \in N$.

Since $\sum_{i \in N} \mathcal{X}_{\eta}^{i}=\sum_{i \in N} \mathcal{X}_{\varsigma}^{i}$, there must be $i_{1} \in N \backslash\left\{i_{0}\right\}$ such that $\mathcal{X}_{\lambda}^{i_{1}} \neq \mathcal{X}_{\varsigma}^{i_{1}}$. As the requirement of strict concavity is violated for at most one agent, the utility function $u_{\sigma}^{i}$ must be strictly concave either for $i=i_{0}$ or for $i=i_{1}$. Thus, inequality (5) is strict for at least one agent in the economy. Hence, an $N$-feasible allocation

1 The function $u_{\sigma}^{i}$ is strongly monotone if the following condition is satisfied: $\left[x_{\sigma}^{i}, y_{\sigma}^{i} \in \mathbb{R}_{+}^{L}, x_{\sigma l}^{i} \geq\right.$ $\left.y_{\sigma l}^{i} \forall l \in\{1, \ldots, L\}, x_{\sigma}^{i} \neq y_{\sigma}^{i}\right]$ implies $\left[u_{\sigma}^{i}\left(x_{\sigma}^{i}\right)>u_{\sigma}^{i}\left(y_{\sigma}^{i}\right)\right]$. 
$y_{\sigma}$ can be constructed in such a way that, when it is substituted for $\widetilde{y}_{\sigma}$ in (5), the inequalities become strict for all $i \in N .^{2}$

Define the consumption stream $\mathcal{Y}^{i}$ of agent $i$ as follows:

$$
\mathcal{Y}_{\eta}^{i}= \begin{cases}\mathcal{X}_{\eta}^{i}, & \text { if } \eta \in \mathbb{D} \backslash\{\lambda, \varsigma\} \\ y_{\sigma}^{i}, & \text { if } \eta=\lambda, \text { or } \eta=\varsigma .\end{cases}
$$

Then the discounted expected utility of the consumption stream $\mathcal{Y}^{i}$ at the initial node is greater than that of the consumption stream $\mathcal{X}^{i}$ for every member $i$ of coalition $N$. Hence, allocation $\mathcal{Y}$ is a utility improving deviation by coalition $N$ from allocation $\mathcal{X}$.

We conclude this section with a characterization of the strong sequential core.

Corollary 1. Let $\mathbf{E}$ be a dynamic exchange economy satisfying all assumptions of Theorem 2. Then

$$
S S C(\mathbf{E})=\left\{\{x\} \mid x \in \bigcap_{s \in \mathbf{S}} C\left(\mathcal{E}_{s}\right)\right\} .
$$

In the special case with only one state of nature the strong sequential core $S S C(\mathbf{E})$ coincides with the classical core $C(\mathbf{E})$. Both the strong sequential core and the classical core consist of those consumption allocations where some distribution of initial endowments $x$ is repeated each period of time, with $x$ being a coreelement of the evaluation economy $\mathcal{E}_{1}$. Thus $S S C(\mathbf{E})$ is non-empty. As Corollary 1 indicates, however, if there are at least two states of nature, and if the cores of the evaluation economies in different states are disjoint, then the strong sequential core is empty. The following example illustrates the point.

\section{An example}

Consider a dynamic exchange economy with two agents called $a$ and $b$, two states $(S=2)$, and a single consumption good in each state $(L=1)$. The instantaneous utility functions are

$$
u_{s}^{i}\left(x_{s}^{i}\right)=\ln \left(x_{s}^{i}\right), i=a, b .
$$

2 The allocation $y_{\sigma}$ can be constructed as follows. Suppose that inequality (5) is strict for agent $i_{0}$. If $\widetilde{y}_{\sigma}^{i_{0}}$ were a zero vector, then strong monotonicity would imply that the inequalities $u_{\sigma}^{i_{0}}\left(\widetilde{y}_{\sigma}^{i_{0}}\right) \leq$ $u_{\sigma}^{i_{0}}\left(\mathcal{X}_{\lambda}^{i_{0}}\right)$ and $u_{\sigma}^{i_{0}}\left(\widetilde{y}_{\sigma}^{i_{0}}\right) \leq u_{\sigma}^{i_{0}}\left(\mathcal{X}_{\varsigma}^{i_{0}}\right)$ hold, contradicting the supposition. Hence, $\widetilde{y}_{\sigma l_{0}}^{i_{0}}$ is non-zero for some good $l_{0} \in\{1, \ldots, L\}$. Let

$$
y_{\sigma l}^{i}= \begin{cases}\widetilde{y}_{\sigma l}^{i}, & \text { if } l \in\{1, \ldots, L\} \backslash\left\{l_{0}\right\}, i \in N, \\ \widetilde{y}_{\sigma l}^{i}-\epsilon & \text { if } l=l_{0}, i=i_{0}, \\ \widetilde{y}_{\sigma l}^{i}+\frac{1}{n-1} \epsilon & \text { if } l=l_{0}, i \in N \backslash\left\{i_{0}\right\} .\end{cases}
$$

Then by strong monotonicity, $u_{\sigma}^{i}\left(y_{\sigma}^{i}\right)>u_{\sigma}^{i}\left(\widetilde{y}_{\sigma}^{i}\right)$ for all $\epsilon>0$ and all $i \in N \backslash\left\{i_{0}\right\}$. Moreover, $\epsilon>0$ can be chosen small enough for the vector $y_{\sigma}^{i_{0}}$ to lie in the consumption set $X_{\sigma}^{i_{0}}$ and to preserve strict inequality in (5). 
For a moment, we leave the matrix of transitional probabilities and the initial endowments unspecified. Aggregate endowment of the consumption good in state $\sigma$ is denoted by $e_{\sigma}^{N}$, and $e^{N} \in \mathbb{R}^{S}$ is the vector of aggregate endowments across all states.

The set of Pareto-efficient allocations of the evaluation economy coincides with the diagonal of the Edgeworth box. That is, distribution $x$ of the initial endowments is Pareto-efficient if agents' consumption bundles $x^{i}$ are proportional to $e^{N}$. For a Pareto-efficient allocation to be in the core of the evaluation economy, the share of the aggregate endowment accrued to each agent should be large enough, so that the conditions of individual rationality hold. Formally,

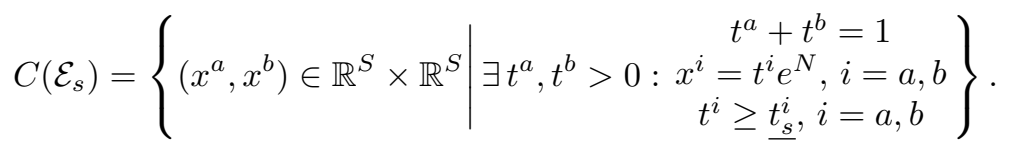

The value for $t_{s}^{i}$ is determined by the condition of individual rationality for agent $i$. It depends on the fundamentals of the dynamic exchange economy as follows:

$$
\underline{t_{s}^{i}}=\prod_{\sigma \in \mathbf{S}}\left(\frac{e_{\sigma}^{i}}{e_{\sigma}^{N}}\right)^{(1-\delta) \psi(\sigma \mid s)} .
$$

Any allocation in the core of the evaluation economy is wholly determined by $t^{a}$, the share of the aggregate endowment enjoyed by agent $a$. In state $s$ the lower value for $t^{a}$ is $t_{s}^{a}$, while the upper value is $1-t_{s}^{b}$. We know (see Corollary 1 ) that the strong sequential core is non-empty if and only if the cores of the evaluation economies in different states have a non-empty intersection. In our example, this is equivalent to the condition that the intervals $\left[t_{1}^{a}, 1-\underline{\left.t_{1}^{b}\right]}\right.$ and $\left[t_{2}^{a}, 1-\underline{t_{2}^{b}}\right]$ overlap.

Figure 2 pictures these intervals for various values of the discount factor $\delta$. To produce this figure the transitional probabilities and the initial endowments were specified as follows:

$$
\pi=\left[\begin{array}{ll}
0.75 & 0.25 \\
0.25 & 0.75
\end{array}\right], e^{a}=\left[\begin{array}{l}
4 \\
1
\end{array}\right], e^{b}=\left[\begin{array}{l}
1 \\
4
\end{array}\right] .
$$

We see that for the values of $\delta$ smaller than, approximately, 0.8 , the cores of the evaluation economies are disjoint, and consequently the strong sequential core is empty. In contrast, for $\delta$ greater than 0.8 the strong sequential core is non-empty. The example therefore suggests that the more patient individuals are, the more likely it is that the strong sequential core is non-empty. The next section validates this suggestion for the general case.

\section{A non-emptiness result}

In this section we consider a family of dynamic exchange economies $\mathbf{E}_{\delta}$ parameterized by a discount factor $\delta \in(0,1)$. We will prove that the strong sequential core of the economy $\mathbf{E}_{\delta}$ is non-empty whenever $\delta$ is sufficiently close to one. 


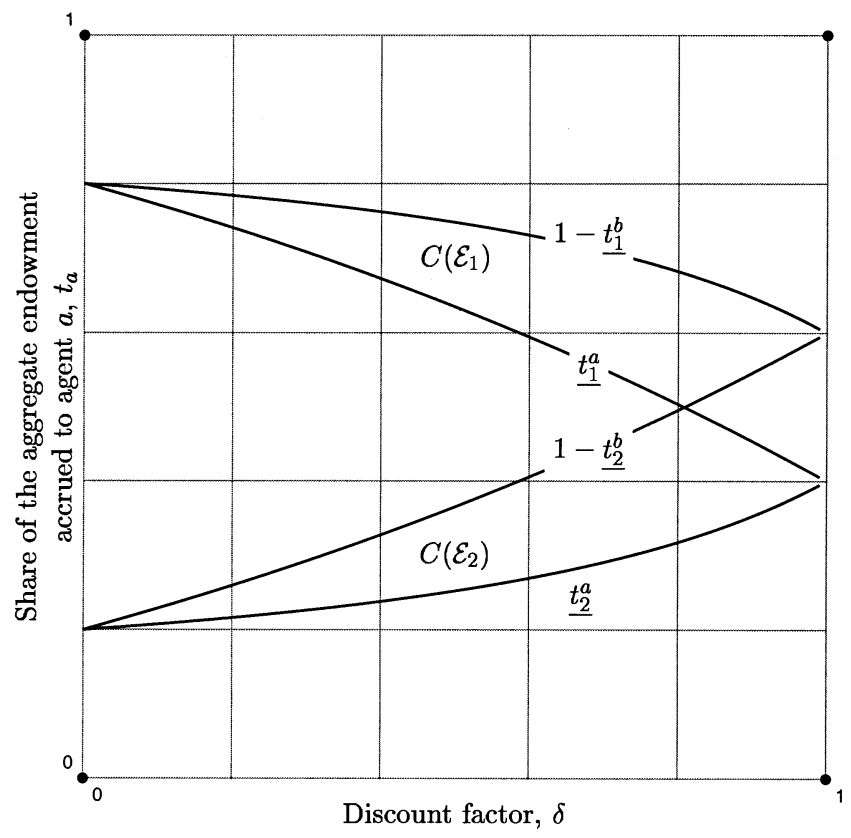

Figure 2. The cores of the evaluation economies

Our approach is to compute the limits for the utility functions $v_{s}^{i}$ as $\delta$ approaches one, and next to construct an economy using the resulting utility functions. This artificial economy will then provide us with some plausible candidates to belong to the strong sequential core of the original dynamic economy.

Throughout this section we write $\psi(\sigma \mid s ; \delta)$ for the elements of the matrix $\psi$ to emphasize their dependence on the discount factor. The utility functions $v_{s}^{i}(\cdot)$ of the evaluation economies $\mathcal{E}_{s}$ depend on the discount factor via $\psi$; we write $v_{s}^{i}(\cdot, \delta)$ and $\mathcal{E}_{s \delta}$.

Clearly, $\psi(\sigma \mid s, \delta)$ explodes as $\delta$ approaches one. When multiplied by $(1-\delta)$, however, it converges to a well-defined limit.

Theorem 3. Suppose that the matrix $\pi$ is irreducible. Then the limit

$$
\phi(\sigma \mid s):=\lim _{\delta \rightarrow 1}(1-\delta) \psi(\sigma \mid s, \delta)
$$

exists for all $\sigma, s \in \mathbf{S}$. Moreover,

(1) the values of $\phi(\sigma \mid s)$ are independent of $s$, i.e. $\phi(\sigma \mid s)=\phi\left(\sigma \mid s^{\prime}\right)$ for all $s, s^{\prime} \in$ $\mathrm{S} \mathbf{;}^{3}$

(2) $\phi(\sigma)>0$ for all $\sigma \in \mathbf{S}$;

(3) the vector $(\phi(\sigma))_{\sigma \in \mathbf{S}}$ is the eigenvector of the matrix $\pi$ corresponding to the eigenvalue $\lambda=1$.

\footnotetext{
3 In what follows we suppress the argument $s$ of $\phi$.
} 
Proof. Let $\lambda_{1}, \ldots, \lambda_{m}(m \leq S)$ be the distinct eigenvalues of the matrix $\pi$.

The matrix $\pi$ is a column stochastic matrix, i.e. its entries in a given column add up to 1 . This implies that $\lambda_{1}=1$ is an algebraically (and hence geometrically) simple eigenvalue of the matrix $\pi$; that the vector $\mathbf{1}=(1, \ldots, 1)^{\top}$ is the eigenvector of the matrix $\pi^{\top}$ corresponding to the eigenvalue $\lambda_{1}$, and that the eigenvector of the matrix $\pi$ corresponding to $\lambda_{1}$ can be set positive.

Let $\pi=T \Lambda T^{-1}$ be the Jordan decomposition of the matrix $\pi$, where $\Lambda$ is a Jordan form. There is a unique Jordan block corresponding to the eigenvalue $\lambda_{1}$ and this block has order 1 . Hence, $\Lambda$ can be written as

$$
\Lambda=\left(\begin{array}{ll}
1 & \mathbf{0} \\
\mathbf{0} & \Gamma
\end{array}\right),
$$

where $\Gamma$ is a direct sum of those Jordan blocks that correspond to eigenvalues $\lambda_{2}, \ldots, \lambda_{m}$. Observe that the matrix $\left(I_{S-1}-\delta \Gamma\right)$ is non-singular for all $\delta \in(0,1) \backslash \bigcup_{j=2}^{m}\left\{\lambda_{j}^{-1}\right\}$. Moreover, the matrix $\left(I_{S-1}-\Gamma\right)$ is non-singular.

The first column of the matrix $T$, denoted $c n_{1} T$ and the first row of the matrix $T^{-1}$, denoted $r w_{1} T^{-1}$ are the eigenvectors of the matrices $\pi$ and $\pi^{\top}$, respectively. We can therefore set

$$
\begin{array}{r}
c n_{1} T>>\mathbf{0} \\
r w_{1} T^{-1}=\alpha \mathbf{1}^{\top}
\end{array}
$$

for some nonzero scalar $\alpha$. Using the Jordan decomposition of the matrix $\pi$ we can write

$$
\begin{array}{r}
\left(I_{S}-\delta \pi\right)^{-1}=T\left(I_{S}-\delta \Lambda\right)^{-1} T^{-1}, \text { where } \\
\left(I_{S}-\delta \Lambda\right)^{-1}=\left[\begin{array}{cc}
(1-\delta)^{-1} & \mathbf{0} \\
\mathbf{0} & \left(I_{S-1}-\delta \Gamma\right)^{-1}
\end{array}\right] .
\end{array}
$$

Since $\left(I_{S-1}-\Gamma\right)$ is invertible,

$$
\lim _{\delta \rightarrow 1}(1-\delta)\left(I_{S}-\delta \Lambda\right)^{-1}=\operatorname{diag}\{1, \mathbf{0}\}
$$

Therefore,

$$
\begin{aligned}
\lim _{\delta \rightarrow 1}(1-\delta)\left(I_{S}-\delta \pi\right)^{-1}=T \operatorname{diag}\{1, \mathbf{0}\} T^{-1}= \\
=c n_{1} T \cdot r w_{1} T^{-1}=\alpha c n_{1} T \cdot \mathbf{1}^{\top} .
\end{aligned}
$$

The following observations complete the proof of Theorem 1:

(1) $\phi(\sigma \mid s)$ is given by the entry $\sigma$ of the vector $\alpha \cdot c n_{1} T$ and therefore is independent of $s$.

(2) $\phi(\sigma \mid s)$ is non-negative, since by definition it is the limit of a positive-valued function. As the vector $c n_{1} T$ has been set positive, Equation (6) implies that $\alpha$ is positive. Hence, $\phi(\sigma \mid s)$ is, in fact, positive.

(3) The vector $\phi=\alpha \cdot c n_{1} T$ is the eigenvector of the matrix $\pi$ corresponding to the eigenvalue $\lambda_{1}$. 
Definition 4. The symbol $\mathcal{E}_{\infty}$ denotes a pure exchange economy in which $\mathbb{R}^{S L}$ is the commodity space, $X^{i}=\prod_{\sigma \in \mathbf{S}} X_{\sigma}^{i}$ is the consumption set, $e^{i}=\left(e_{\sigma}^{i}\right)_{\sigma \in \mathbf{S}}$ is the vector of initial endowments, and $\vartheta^{i}: X^{i} \mapsto \mathbb{R}$ is the utility function defined by

$$
\vartheta^{i}\left(x^{i}\right)=\sum_{\sigma \in \mathbf{S}} \phi(\sigma) u_{\sigma}^{i}\left(x_{\sigma}^{i}\right)
$$

Theorem 4. Let $\mathbf{E}_{\delta}$ be a family of dynamic exchange economies, satisfying assumption $\mathbf{A}$, in which the instantaneous utility functions $u_{s}^{i}$ are strictly concave and locally non-satiated for all $i \in N$, and $s \in \mathbf{S}$. Let $\bar{x}$ be an equilibrium allocation of the economy $\mathcal{E}_{\infty}$. Then there exists a $\bar{\delta} \in(0,1)$ such that $\{\bar{x}\} \in S S C\left(\mathbf{E}_{\delta}\right)$ for all $\delta \in(\bar{\delta}, 1)$.

To prove Theorem 4 we first establish an auxiliary lemma. Let

$$
\begin{aligned}
& X_{\sigma}(Q)=\left\{x_{\sigma} \in \prod_{i \in Q} X_{\sigma}^{i} \mid \sum_{i \in Q} x_{\sigma}^{i}=\sum_{i \in Q} e_{\sigma}^{i}\right\} \\
& X(Q)=\prod_{\sigma \in \mathbf{S}} X_{\sigma}(Q) .
\end{aligned}
$$

Let $\mathcal{P}_{Q}\left(\mathcal{E}_{\infty}\right)$ denote the set of weakly efficient allocations for a coalition $Q$ in the economy $\mathcal{E}_{\infty}$. Recall that $x \in \mathcal{P}_{Q}\left(\mathcal{E}_{\infty}\right)$ if and only if $x \in X(Q)$ and there is no $y \in X(Q)$ such that $\vartheta^{i}\left(y^{i}\right)>\vartheta^{i}\left(x^{i}\right)$ for all $i \in Q$. The set $\mathcal{P}_{Q}\left(\mathcal{E}_{s \delta}\right)$ is similarly defined for the evaluation economy $\mathcal{E}_{s \delta}$.

Lemma 2. Let $\mathbf{E}_{\delta}$ be a family of dynamic exchange economies satisfying assumption A. Then $\mathcal{P}_{Q}\left(\mathcal{E}_{\infty}\right)=\mathcal{P}_{Q}\left(\mathcal{E}_{s \delta}\right)$ for all $Q \subseteq N, s \in \mathbf{S}$ and $\delta \in(0,1)$.

Proof. An allocation $x$ is an element of $\mathcal{P}_{Q}\left(\mathcal{E}_{\infty}\right)$ if and only if there exist weights $\alpha^{i} \geq 0(i \in Q), \quad \sum_{i \in Q} \alpha^{i}=1$, such that $x$ maximizes the function

$$
\sum_{i \in Q} \alpha^{i} \vartheta^{i}\left(x^{i}\right)=\sum_{\sigma \in \mathbf{S}} \phi(\sigma) \sum_{i \in Q} \alpha^{i} u_{\sigma}^{i}\left(x_{\sigma}^{i}\right)
$$

over the set $X(Q)$. Since the values of $\phi(\cdot)$ are positive, this is equivalent to the requirement that $x_{\sigma}$ maximizes the function

$$
\sum_{i \in Q} \alpha^{i} u_{\sigma}^{i}\left(x_{\sigma}^{i}\right)
$$

over $X_{\sigma}(Q)$ for all $\sigma \in \mathbf{S}$. Again, since the values of $\psi(\cdot)$ are positive, this is equivalent to the condition that $x$ maximizes the function

$$
\sum_{\sigma \in \mathbf{S}} \psi(\sigma \mid s, \delta) \sum_{i \in Q} \alpha^{i} u_{\sigma}^{i}\left(x_{\sigma}^{i}\right)=\sum_{i \in Q} \alpha^{i} v_{s}^{i}\left(x^{i}, \delta\right)
$$

over $X(Q)$. This last condition is equivalent to $x \in \mathcal{P}_{Q}\left(\mathcal{E}_{s \delta}\right)$. 
Proof of Theorem 4. We have to show that for all values of $\delta$ sufficiently close to one and for all $s \in \mathbf{S}$, the allocation $\bar{x}$ is in the core of the economy $\mathcal{E}_{s \delta}$ (see Theorem 1 ). Suppose that this is not the case. Then there exist a state $s \in \mathbf{S}$, coalition $Q \subseteq N$, sequences $\delta_{m} \in(0,1)$ and $x_{(m)} \in X(Q)$ such that

$$
\begin{array}{r}
\lim \delta_{m}=1, \\
v_{s}^{i}\left(x_{(m)}^{i} ; \delta_{m}\right)>v_{s}^{i}\left(\bar{x}^{i} ; \delta_{m}\right) \quad \forall i \in Q .
\end{array}
$$

Since $X(Q)$ is a compact set, there is a subsequence of $x_{(m)}$ converging to an element $x$ of $X(Q)$. Premultiplying inequality (7) by $\left(1-\delta_{m}\right)$, replacing sequences by subsequences and taking the limit yields

$$
\vartheta^{i}\left(x^{i}\right) \geq \vartheta^{i}\left(\bar{x}^{i}\right), \quad \forall i \in Q .
$$

Let $\bar{p}$ be a vector of prices corresponding to equilibrium allocation $\bar{x}$. Under the assumption of local non-satiation of the instantaneous utility functions, inequalities (8) imply that

$$
\bar{p} x^{i} \geq \bar{p} e^{i}, \quad \forall i \in Q
$$

If there were some strict inequality in (9), then $x$ would not be feasible for coalition $Q$. Hence,

$$
\bar{p} x^{i}=\bar{p} e^{i}, \quad \forall i \in Q
$$

The strict concavity assumption implies that there exists a unique maximizer of the utility function $\vartheta^{i}$ in the budget set at prices $\bar{p}$, i.e.

$$
x^{i}=\bar{x}^{i}, \quad \forall i \in Q .
$$

Hence, $\left(\bar{x}^{i}\right)_{i \in Q} \in X(Q)$. This implies that the allocation $\left(\bar{x}^{i}\right)_{i \in Q}$ is not only feasible, but also weakly efficient for the members of the coalition $Q$ in the economy $\mathcal{E}_{\infty}$. According to Lemma 2 , however, the set of weakly efficient allocations for any coalition in the economy $\mathcal{E}_{\infty}$ coincides with that in the economy $\mathcal{E}_{s \delta}$ for all $s \in \mathbf{S}$ and $\delta \in(0,1)$. Therefore, $\left(\bar{x}^{i}\right)_{i \in Q}$ is weakly efficient for $Q$ in the economies $\mathcal{E}_{s \delta}$ as well. This contradicts, however, the original assumption.

It remains to give explicit assumptions that will guarantee existence of an equilibrium for the economy $\mathcal{E}_{\infty}$. We require that the instantaneous utility functions be strongly monotone and that the aggregate endowments of commodities be positive.

Corollary 2. Let $\mathbf{E}_{\delta}$ be a family of dynamic exchange economies, satisfying assumption $\mathbf{A}$, in which the instantaneous utility functions $u_{s}^{i}$ are strictly concave and strongly monotone for all $i \in N$ and $s \in \mathbf{S}$, and $\sum_{i \in N} e_{s l}^{i}>0$ for all $l \in\{1, \ldots, L\}$ and $s \in \mathbf{S}$. Then there exists a $\bar{\delta} \in(0,1)$ such that $S S C\left(\mathbf{E}_{\delta}\right) \neq \emptyset$ for all $\delta \in(\bar{\delta}, 1)$. 
An example below shows that a violation of the strict concavity assumption in Corollary 2 may result in the strong sequential core being empty for all values of the discount factor.

Example. Consider a family $\mathbf{E}_{\delta}$ of dynamic exchange economies with three agents called $a, b$, and $c$, two states $(S=2)$, and a single consumption good in each state $(L=1)$. The state-independent instantaneous utility functions are

$$
\begin{aligned}
& u_{\sigma}^{i}\left(x_{\sigma}^{i}\right)=\ln \left(x_{\sigma}^{i}\right), \quad x_{\sigma}^{i}>0, \quad i=a, b, \\
& u_{\sigma}^{c}\left(x_{\sigma}^{c}\right)=x_{\sigma}^{c}, \quad x_{\sigma}^{c} \geq 0 .
\end{aligned}
$$

Notice that the function $u_{\sigma}^{c}$ is not strictly concave, thus violating one of the assumptions of Corollary 2. The initial endowments and the transition probabilities are specified as follows:

$$
e^{a}=\left[\begin{array}{l}
4 \\
1
\end{array}\right], e^{b}=\left[\begin{array}{l}
1 \\
4
\end{array}\right], e^{c}=\left[\begin{array}{l}
5 \\
5
\end{array}\right], \pi=\left[\begin{array}{ll}
0.25 & 0.25 \\
0.75 & 0.75
\end{array}\right] .
$$

The core of the evaluation economy is then a single-element set given by

$$
C\left(\mathcal{E}_{s \delta}\right)=\left\{\left[\begin{array}{lll}
\kappa_{s \delta}^{a} & \kappa_{s \delta}^{b} & 5 \\
\kappa_{s \delta}^{a} & \kappa_{s \delta}^{b} & 5
\end{array}\right]\right\}
$$

where the values for $\kappa_{s \delta}^{i}$ depend on the fundamentals of the economy $\mathbf{E}_{\delta}$ as follows:

$$
\kappa_{s \delta}^{i}=(1-\delta) \sum_{\sigma \in \mathbf{S}} \psi(\sigma \mid s ; \delta) e_{\sigma}^{i} .
$$

It is easy to check that $\kappa_{1 \delta}^{a} \neq \kappa_{2 \delta}^{a}$, implying that $C\left(\mathcal{E}_{1 \delta}\right) \bigcap C\left(\mathcal{E}_{2 \delta}\right)=\emptyset$ for all $\delta \in(0,1)$. Observe that the economies $\mathbf{E}_{\delta}$ satisfy all assumptions of Theorem 2 . Corollary 1 therefore implies that the strong sequential core of the economy $\mathbf{E}_{\delta}$ is empty for all $\delta \in(0,1)$.

\section{Concluding remarks}

To conclude, we point out some limitations and suggest a number of extensions of the approach taken in this paper.

We consider an economy with infinite time horizon where at each moment of time the state reveals and determines the instantaneous utility functions and initial endowments at that moment. The state follows a Markov process. Contrary to the stochastic-games literature we assume that the transitional probabilities are entirely exogenous in the sense that they cannot be influenced by the agent's consumption decisions. This makes a sub-economy starting at a particular point in time independent of the commodity allocation chosen up to that point. Thus the dynamic links between the time periods are rather limited.

While this may seem a restrictive assumption, it is not obvious exactly how the decisions of the players or coalitions should influence the transitional probabilities, given the cooperative nature of the economy in question. The study of dynamic 
economies with endogenous transitional probabilities is a subject of further research.

Neither can the agents influence future sub-economies by trading assets. Indeed, the assumption of the model is that contracts allowing for intertemporal trade are not enforceable. This should be seen as an extreme case of market incompleteness. As the study of two-period economies indicates, however (see Predtetchinski, Herings, and Peters, 2002), the strong sequential core is weakly increasing in the number of enforceable securities. Thus, for the purpose of obtaining the non-emptiness result, the case where there are no securities is the most difficult one.

The possible emptiness of the strong sequential core calls for an examination of weaker solution concepts. In the weak sequential core it is required that the commodity allocation be robust only against those deviations which cannot be improved upon by any sub-coalition of the deviating coalition. Thus, the number of deviations to be considered is reduced, and a weakening of the strong sequential core is obtained. The weak sequential core for dynamic games with transferable utility is studied in Kranich, Perea, and Peters (2001), and for two-period exchange economies in Predtetchinski, Herings, and Perea (2002). The translation of the weak sequential core concept into the present framework is an interesting problem for future research.

\section{References}

Aumann, R. J.: The core of a cooperative game without side payments. Transactions of the American Mathematical Society 98, 539-552 (1961)

Filar, J.A., Petrosjan, L. A.: Dynamic cooperative games. International Game Theory Review 2, 47-65 (2000)

Gale, D.: The core of a monetary economy without trust. Journal of Economic Theory 19, 456-491 (1978)

Koutsougeras, L. C.: A two-stage core with applications to asset market and differential information economies. Economic Theory 11, 563-584 (1998)

Koutsougeras, L. C., Yannelis, N. C.: Bounded rational learning in differential information economies: core and value. Journal of Mathematical Economics 31, 373-391 (1999)

Kranich, L., Perea, A., Peters, H.: Core concepts for dynamic TU-game. METEOR Discussion Paper 01/024, Universiteit Maastricht, pp. 1-25 (2001)

Predtetchinski, A., Herings, P. J. J., Peters, H.: The strong sequential core for two-period economies. Journal of Mathematical Economics 38, 465-482 (2002)

Predtetchinski, A., Herings, P. J. J., Perea, A.: The weak sequential core for two-period economies. METEOR Discussion Paper 02/011, Universiteit Maastricht, pp. 1-30 (2002)

Repullo, R.: The core of an economy with transaction costs. Review of Economic Studies 55, 447-458 (1988)

Serfes, K.: Non-myopic learning in differential information economies: the core. Economic Theory $\mathbf{1 8}$, 333-348 (2001) 